Scientia Marina 82(2)

June 2018, 95-106, Barcelona (Spain)

ISSN-L: 0214-8358

https://doi.org/10.3989/scimar.04781.14A

\title{
Free-diving underwater fish photography contests: a complementary tool for assessing littoral fish communities
}

\author{
Ana Gordoa ${ }^{1}$, Jordi Boada ${ }^{1}$, Antoni García-Rubies ${ }^{1}$, Oscar Sagué ${ }^{2}$ \\ ${ }^{1}$ Department of Marine Ecology. Centro de Estudios Avanzados de Blanes, Spanish National Research Council (CSIC), \\ Blanes, Girona, Spain. \\ (AG) (Corresponding author) E-mail: gordoa@ceab.csic.es. ORCID iD: http://orcid.org/0000-0003-1822-8196 \\ (JB) E-mail: jboada@ ceab.csic.es. ORCID iD: http://orcid.org/0000-0002-3815-625X \\ (AG-R) E-mail: tonigr@ceab.csic.es. ORCID iD: http://orcid.org/0000-0002-4824-9337 \\ ${ }^{2}$ International Forum for Sustainable Underwater Activities (IFSUA), Carrer València 231, Bxs., Ap. correus 36003 , 08007 \\ Barcelona, Spain. \\ (OS) E-mail: ifsua@ifsua.net. ORCID iD: http://orcid.org/0000-0002-4053-6614
}

\begin{abstract}
Summary: Characterizing fish communities must be a priority to safeguard resources and determine critical changes. Here, species richness and the spatial and temporal evolution in the structure of fish assemblages were analysed based on photos taken in underwater free-diving contests. A total of 29 contests held from 2008 to 2015 at four different locations along the northeastern Spanish coast, including a marine protected area were analysed. Contests reward the number of species per participant and photographic quality. Species image frequency from each tournament were standardized to catch image rate. A total of 88 taxa were recorded, including 32 cryptobenthic species, the highest number recorded in the Mediterranean littoral system so far. Cluster analyses yielded four major groups. Catch image rates in the marine protected area were significantly higher for seven species of high commercial interest and for two big labrids of recreational interest, including an endangered species (Labrus viridis). Overall, the study showed that photographic free-diving contest data are a potential tool for determining species richness in littoral systems since contest rules promote competition between participants to obtain maximum fish diversity. We believe that this type of cost-effective data can be applied worldwide as a complementary way of monitoring littoral fish assemblage.
\end{abstract}

Keywords: littoral fish assemblages; species richness; diversity; photography contests; Mediterranean Sea.

Competiciones fotográficas de peces en buceo libre: una herramienta complementaria para la valoración de las comunidades de peces litorales

Resumen: La caracterización de las comunidades de peces debe ser una prioridad para salvaguardar los recursos y para la detección de cambios críticos. La información de las competiciones fotográficas a buceo libre se utilizó para analizar la riqueza específica y las variaciones espaciales y temporales de la comunidad íctica. Se analizaron un total de 29 concursos celebrados entre el 2008 y el 2015 en cuatro puntos de la costa Noroeste de España, incluida un área marina protegida. Estas competiciones premian a los participantes por el número de especies fotografiadas y su calidad. En cada concurso se estandarizó la frecuencia de imágenes por especie, convirtiéndola a la tasa de imágenes capturadas. Se registraron 88 taxones, incluidas 32 especies criptobénticas, el mayor número registrado hasta la fecha en el litoral Mediterráneo. El análisis de conglomerados identificó cuatro grupos principales. La tasa de imágenes capturadas de siete especies comerciales fue superior en el AMP y también superior para dos especies de lábridos de interés recreativo, una de ellas considerada como especie amenazada (Labrus viridis). En suma, el estudio mostró que los datos de los concursos fotográficos a buceo libre son una herramienta potencial para determinar la riqueza específica en los sistemas litorales debido a que las competiciones fomentan la competencia entre los participantes para obtener la máxima diversidad de especies. Consideramos que estos datos, eficaces en los costes, se pueden utilizar a nivel mundial para complementar los sistemas de seguimiento de las comunidades de peces litorales.

Palabras clave: comunidades de especies litorales; riqueza específica; diversidad; concursos fotográficos, mar Mediterráneo.

Citation/Cómo citar este artículo: Gordoa A., Boada J., García-Rubies A., Sagué O. 2018. Free-diving underwater fish photography contests: a complementary tool for assessing littoral fish communities. Sci. Mar. 82(2): 95-106. https://doi. org/10.3989/scimar.04781.14A

Editor: E. Macpherson.

Received: March 20, 2018. Accepted: June 11, 2018. Published: June 20, 2018.

Copyright: (C) 2018 CSIC. This is an open-access article distributed under the terms of the Creative Commons Attribution 4.0 International (CC BY 4.0) License. 


\section{INTRODUCTION}

The marine coastal environment is threatened both locally and globally in different ways. In Europe the coastal habitats have been progressively degraded over the last few decades (e.g. Benedetti-Cecchi et al. 2001, Lotze et al. 2006, Claudet and Fraschetti 2010). Future projections are not encouraging: The Mediterranean Action Plan predicts that the urban population of the coastal Mediterranean could reach 176 million by 2015 plus 350 million tourists yearly. Furthermore, an increase in the number of introduced species (Boudouresque et al. 2005) and climate-induced stressors are contributing to changes in the Mediterranean biodiversity (Fraschetti et al. 2011), emphasizing the need to foster the surveillance of coastal systems.

Maintaining diversity in coastal systems is essential for the sustainability of communities, ecosystem functioning and services (Pickaver 2009, Costanza et al. 2014). Changes in coastal ecosystems can be oneoff events and have dramatic effects (i.e. artificial modifications of the coast or severe storms), or rather progressive and relatively slow (at the scale of human life), and are difficult to notice through snapshot studies. Moreover, the negative impact on them may be difficult to document (Støttrup 2009). One of the aims of the European Marine Directive is to ensure that biodiversity is maintained, that is, kept in line with the natural state appropriate to the area in question. However, baseline shifts can affect reference sites as much as impact sites (Støttrup 2009), masking variations and distorting perceptions.

In the Mediterranean, littoral fish communities are threatened by numerous stressors, including habitat loss, global warming, changes in the continental water discharges, artificialization of the coastlines, introduction of alien species and fishing pressure (e.g. Guidetti et al. 2002, Claudet et al. 2006). Fish communities are a key component of the aquatic ecosystem (Holmlund and Hammer 1999) as fish provide fundamental services for ecosystem functioning and resilience (Schindler et al. 1997, Vanni 2002, Myers et al. 2007). Moreover, numerous studies support the use of fish assemblages as biological indicators for marine coastal waters (e.g. Sano 2000, Seytre and Francour 2008, Azzurro et al. 2011). Nevertheless, studying and monitoring fish assemblages is as complex as the diversity of groups represented. Littoral fish assemblages include groups of different niches, so different complementary sampling methods are required to capture all the components (Elliott et al. 2002). It is widely recognized that no single assessment technique can provide unbiased, qualitative or quantitative estimates of fish assemblages (Sale and Douglas 1981, Ackerman and Bellwood 2000). This problem was highlighted decades ago (HarmelinVivien et al. 1985) but it is still an open issue and it is recommended to apply different sampling methods simultaneously to the same study site (Connell et al. 1998, Willis et al. 2000, Cappo et al. 2004).

Studies of fish populations in shallow littoral waters usually rely on underwater visual censuses (e.g. Harmelin-Vivien et al. 2008, Bussotti et al. 2015,
Prato et al. 2017). Video techniques have been increasingly used (Mallet and Pelletier 2014) and are recognized mainly as an additional technique to underwater visual censuses, with stationary (Francour 1999), diver-operated (Tessier et al. 2013) or roving cameras (Tessier and Chabanet 2006), or stationary baited underwater video (Gledhill et al. 1996, Willis and Babcock 2000, Stobart et al. 2007). Fishing techniques are also used as a complementary method in littoral fish research surveys (Franco et al. 2012). The appropriateness of a methodological approach depends on its capacity to fulfil the purposes of the study. However, accurate standardized measurements of fish species richness and community structure are essential for monitoring the progress towards biodiversity targets (Hutchings and Baum 2005), as well as for conservation actions (MacNeil et al. 2008). Underwater visual census methods cannot assume equal detectability across all species, and it is recognized that this methodology under-represents a large number of cryptobenthic fish species (e.g. Smith 1988, Ackerman and Bellwood 2000, Kovačić et al. 2012) because more than $90 \%$ of them may go undetected by underwater visual censuses (Willis 2001). In fact, cryptic fish are particularly difficult to accurately survey. They are under-sampled components of fish communities and their ecological roles have generally been ignored (Smith-Vaniz et al. 2006). The use of destructive techniques such as ichthyocides and anaesthetics for cryptic species is controversial but also justified for obtaining reasonably complete inventories of reef fish (Smith-Vaniz et al. 2006, Glavičić et al. 2016, Thiriet et al. 2016).

In addition to research surveys, fisheries-dependent data are also used for fish assemblage monitoring. For all their flaws and lack of scientific methodology, fisheries-dependent data have some advantages: they generally provide large data sets with wide temporal and spatial coverage and they are cost-effective. Furthermore, other cost-effective sources of information are providing good findings in different fields (http:// www.observadoresdelmar.es, Fairclough et al. 2014) jointly with citizen science (e.g. www.seawatchers. org, redmap.org.au), which is currently expanding worldwide. Valuable data gathered by public agencies or private entities also have informative potential that is worthy of being analysed. This is the case of recreational fishing tournaments in certain regions, which have extensive spatial coverage and/or temporal continuity, and have been shown to be valuable for characterizing and monitoring littoral fish assemblages (Lincoln Smith 1989, Coll et al. 2004, Gordoa 2009).

Another potential source of information is underwater photo contests. The technological developments in underwater photography have led to the expansion of a new sport activity: free-diving underwater photography. A recent event in underwater fish photography contests rewards the number of species photographed. Participants are given scores according to the number of species photographed, and they therefore search in every possible habitat to obtain the largest possible number of species. Consequently, the species richness 
resulting from these tournaments is expected to be higher than when traditional visual survey techniques are used. In Spain, and particularly in Catalonia, these tournaments began in the 1980s and have now become regular. The objective of this study was to assess species richness and classify fish communities based on the underwater free-diving fish photography contests held in Catalonia at four different locations from 2008 to 2015 , including a no-take marine protected area (Medes Islands marine reserve).

\section{MATERIALS AND METHODS}

\section{Data description}

This study analysed the information from the freediving photography contests held in Catalonia, which was provided by the Catalan Underwater Activities Federation (FECDAS). The objective of these contests is to photograph only of live fish in the natural system while free-diving. The participants aim to photograph the highest number of fish species possible. Each participant can only present one photo per species so the number of photos per participant is equivalent to the number of species per participant. In Catalonia, the first official championship was held in 2005, and since then FECDAS has gathered the data from each contest, which includes the following information: date, location, number of participants, number of species and total number of photographs per species. In this study we only considered the championships held after 2007 when analogue cameras were completely replaced by digital ones.

A total of 29 tournament reports were analysed. Each tournament had on average 18 participants and lasted 5 hours. FECDAS provided us with a copy of the photographic database, which allowed us to validate the records of dubious species appearing in the contest reports.

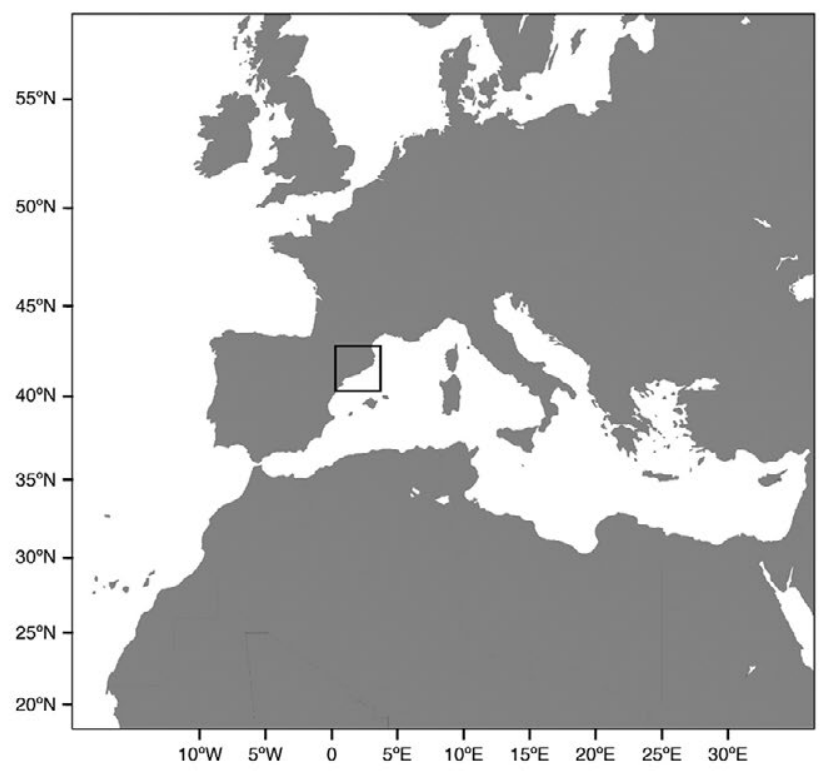

\section{Site features}

The contests were held at four locations along the Catalan coast in northeast Spain (Fig. 1). Annual contests are held at the same locations and provide informative data for studying the spatial and annual variability of littoral fish communities. In addition to their latitudinal differences, the four locations also have different habitat features, and these differences should also be reflected in their fish assemblages.

The Medes Islands, located in the northern area, are a group of islands $\sim 1 \mathrm{~km}$ from the mainland coast. This archipelago is one of the oldest marine reserves in the western Mediterranean. It has been protected since 1983 and has become a popular scuba diving destination. The contests were located on the southern side of the main island, at depths ranging from 0 to $20 \mathrm{~m}$ and characterized by diverse habitats: rocks, sand, Posidonia oceanica meadow and coralligenous. The second location, also in the northern area and near to the Medes Marine Reserve, was a coastal zone of Palamós, Margarida Cove, which has a diverse habitat: rocks, breakwater blocks, sand and $P$. oceanica meadow, at depths ranging from 0 to $15 \mathrm{~m}$. The third location was Mataró, where the contest site was located $~ 500 \mathrm{~m}$ offshore from the coast at depths ranging from 6 to $12 \mathrm{~m}$. The location is restricted to a natural rock barrier that is over $150 \mathrm{~m}$ long, has many cracks and cavities, and is surrounded by sandy beds with some $P$. oceanica patches at the eastern extreme. Finally, the fourth location was L'Ametlla, a coastal area at Port d'Estany, which has brackish water of depths between 0 and 10 $\mathrm{m}$ with a similar bottom structure: rocks, sand and a large $P$. oceanica meadow.

\section{Data analysis}

Species relative abundance data from the tournament information were estimated from the catch image

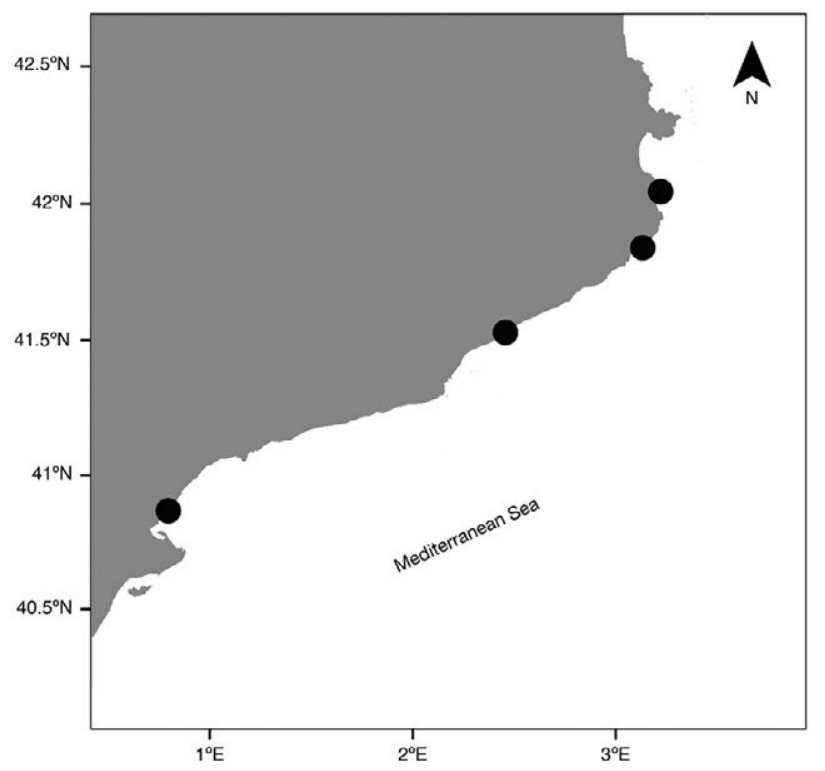

Fig. 1. - Study area and locations of free-diving underwater fish photography. A, Medes Islands; B, Palamós; C, Mataró; and D, Ametlla de Mar. 
Table 1. - Descriptors of the free-diving fish photograph contests studied.

\begin{tabular}{|c|c|c|c|c|c|}
\hline Location & Date & No. photos & No. species & No. participants & No. photos/participants \\
\hline Ametlla de Mar & $30 / 05 / 2009$ & 574 & 61 & 15 & 38 \\
\hline Ametlla de Mar & $16 / 06 / 2012$ & 603 & 62 & 20 & 30 \\
\hline Ametlla de Mar & $26 / 05 / 2013$ & 355 & 52 & 13 & 27 \\
\hline Ametlla de Mar & $08 / 06 / 2014$ & 478 & 68 & 13 & 37 \\
\hline Ametlla de Mar & $31 / 05 / 2015$ & 315 & 67 & 7 & 45 \\
\hline Mataró & $17 / 05 / 2009$ & 452 & 46 & 14 & 32 \\
\hline Mataró & $23 / 05 / 2010$ & 635 & 47 & 24 & 26 \\
\hline Mataró & $22 / 05 / 2011$ & 537 & 47 & 22 & 24 \\
\hline Mataró & $20 / 05 / 2012$ & 337 & 42 & 13 & 26 \\
\hline Mataró & $09 / 06 / 2013$ & 277 & 48 & 9 & 31 \\
\hline Mataró & $18 / 05 / 2014$ & 449 & 53 & 15 & 30 \\
\hline Mataró & $17 / 05 / 2015$ & 289 & 47 & 9 & 32 \\
\hline Medes & $18 / 03 / 2007$ & 251 & 49 & 9 & 28 \\
\hline Medes & $05 / 04 / 2008$ & 560 & 58 & 20 & 28 \\
\hline Medes & $04 / 04 / 2009$ & 650 & 62 & 21 & 31 \\
\hline Medes & $17 / 04 / 2010$ & 1001 & 62 & 34 & 29 \\
\hline Medes & $09 / 04 / 2011$ & 931 & 63 & 31 & 30 \\
\hline Medes & $22 / 04 / 2012$ & 536 & 57 & 20 & 27 \\
\hline Medes & $22 / 06 / 2013$ & 917 & 70 & 22 & 42 \\
\hline Medes & $21 / 06 / 2014$ & 1147 & 78 & 24 & 48 \\
\hline Medes & $20 / 06 / 2015$ & 389 & 66 & 9 & 43 \\
\hline Palamós & $27 / 05 / 2007$ & 368 & 52 & 13 & 28 \\
\hline Palamós & $18 / 05 / 2008$ & 627 & 62 & 21 & 30 \\
\hline Palamós & $22 / 03 / 2009$ & 469 & 60 & 16 & 29 \\
\hline Palamós & $07 / 03 / 2010$ & 692 & 54 & 28 & 25 \\
\hline Palamós & $03 / 06 / 2012$ & 669 & 56 & 20 & 33 \\
\hline Palamós & $12 / 05 / 2013$ & 641 & 59 & 18 & 36 \\
\hline Palamós & $04 / 05 / 2014$ & 686 & 62 & 18 & 38 \\
\hline Palamós & $03 / 05 / 2015$ & 472 & 65 & 11 & 43 \\
\hline
\end{tabular}

rate for each location, which was estimated as the total number of reported photos per species divided by the total number of participants. Taxonomic resolution was not fully attained and some species were classified at the level of genera (Atherina and Sphyraena) or family (Mugilidae).

Absolute number of species richness (S) and Margalef index, $\alpha=(\mathrm{S}-1) / \ln (\mathrm{N})$ ( $\mathrm{N}$ total number of images) were estimated as measures of species richness and diversity for each contest. One-way ANOVAs using STATISTICA 10 (Stat-Soft Inc. 2010) were performed to assess potential differences in species richness and diversity $(\alpha)$ among locations. As the number of samples varies among localities the weighted mean was used in the analysis. The spatial structure and fish community was analysed using the PRIMER software package (Clarke KR and tutorial. PRIMER-E) and agglomerative hierarchical clustering was applied. A community similarity matrix based on the Pearson correlation coefficient was calculated for hierarchical group average linking. Catch rates were not transformed because participants could only present a single photo per species, regardless of the number of photos or encounters they had with the most frequent species. Consequently, the most frequent species were already down-weighted by the contest rules, but the information of species relative frequency was retained because it is equivalent to the probability of being photographed.

A silhouette analysis (Rousseeuw 1987) was performed to evaluate the precision of the hierarchical clustering using the $\mathrm{R}$ statistical software (R Development Core Team, 2013). Silhouette analyses were performed to evaluate consistency within the identified clusters. Differences were examined with an analysis of the similarity percentages (SIMPER). This procedure used to identify which species contributed to the resulting clusters. The analysis was complemented by multidimensional scaling (MDS).

One-way ANOVA was used to assess catch image rate differences between locations in the species of commercial interest. Unequal N HSD post hoc tests (multiple comparison test), a modification of the Tukey test for unequal sample sizes (Winer et al. 1991), were used to determine the significant differences to investigate the efficacy of free-diving photographic data to detect the already known effect of this marine protected area (MPA).

\section{RESULTS}

In total, 16307 photographs corresponding to 29 different contests were analysed. The mean number of participants per contest was 17 and the average number of photographs per participant was 33. The number of photographs per participant has increased over recent years at two locations, Palamós and the Medes Islands, with no effect on the total number of species recorded (Table 1).

A total of 88 taxa belonging to 26 families were photographed (Table 2). The actual species richness was higher, as the genera Atherina, Sphyraena, Trachurus and Trachinus and the family Mugilidae were not identified to the species level. The families with the highest number of species were Sparidae with 17 species and Labridae with 15 species, followed by Blenniidae and Gobiidae with 14 and 10 species, respectively. According to the International Union for Conservation of Nature (IUCN) Red List categories and criteria, 2 of these species are not yet evaluated, 1 is data-deficient to assess its status, 80 are on the least-concern list, 3 are considered vulnerable, 1 is near threatened endangered and 1 is already endangered. It is worth mentioning that 

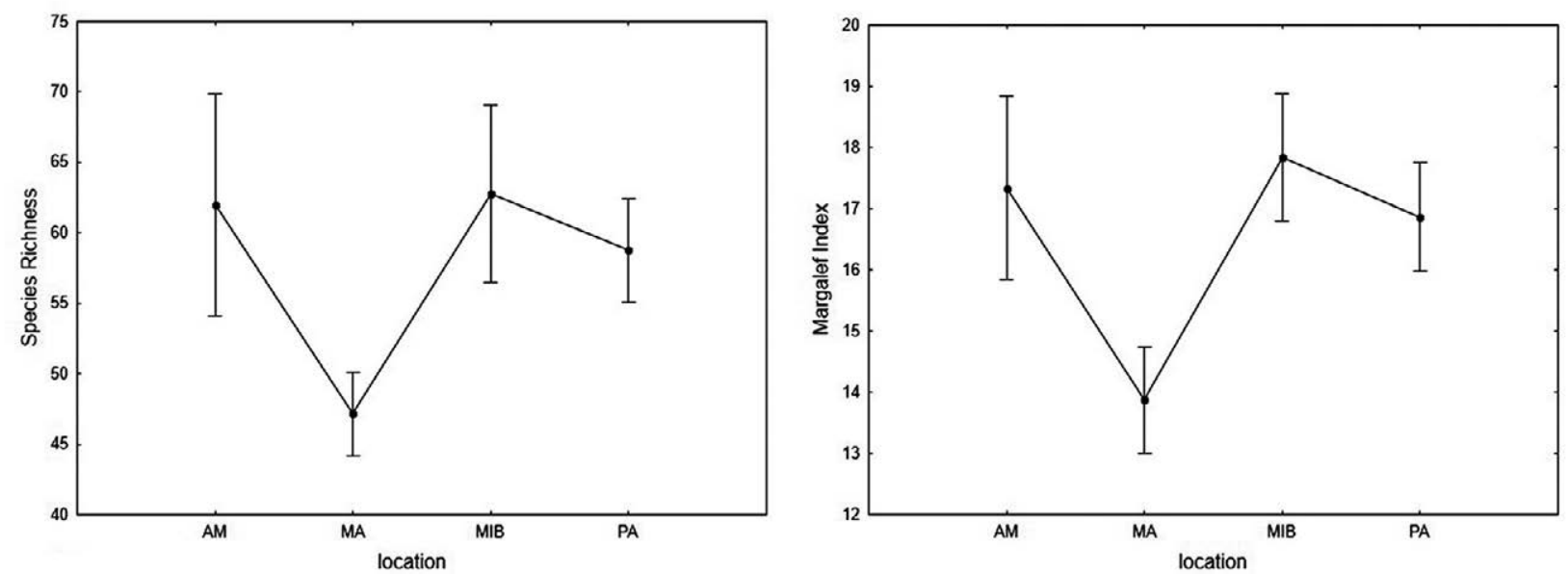

Fig. 2. - Weighted mean and 0.95 confidence intervals of species richness and Margalef diversity index per location. AM, Ametlla de Mar ( $\mathrm{N}=5)$; MA, Mataró (N=7); MIB, Medes Islands (N=9); and PA, Palamós (N=8).

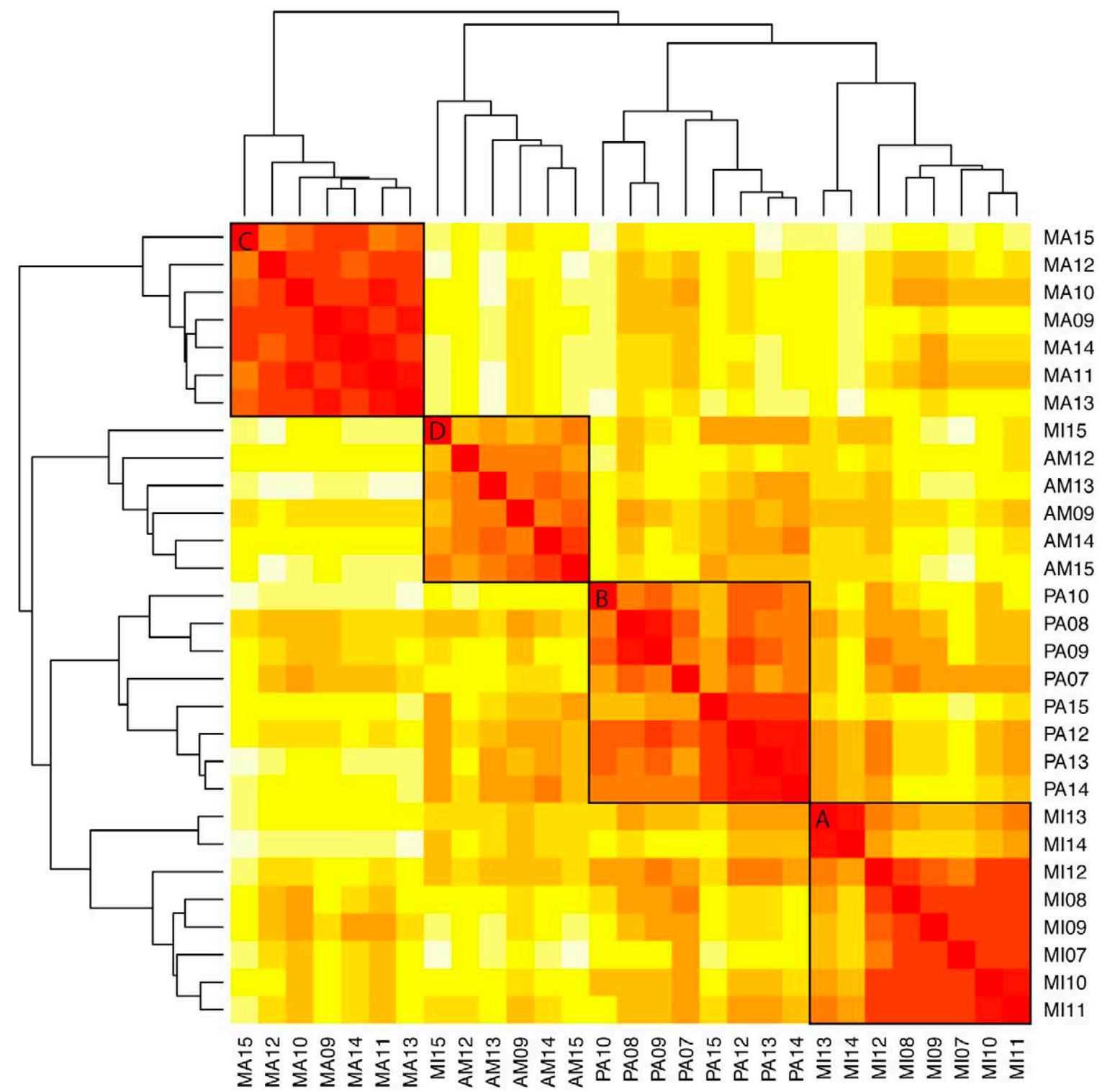

Fig. 3. - Heatmap result of cluster analysis of locations on the basis of species images catch rates. A, B, C and D represent the four main groups identified in the analysis. 
$100 \cdot$ A. Gordoa et al.

Table 2. - Fish species average catch image rate and standard deviation by location. The letters within brackets next to the species correspond to those with a catch image rate significantly higher in the Medes Marine Reserve; each letter corresponds to the location where the significant differences were observed. Asterisks indicate a species described for the first time in the Reserve.

\begin{tabular}{|c|c|c|c|c|c|c|c|c|c|c|c|c|}
\hline \multirow{2}{*}{ Species } & \multirow{2}{*}{$\begin{array}{l}\text { Feeding } \\
\text { (trophic } \\
\text { levels) }\end{array}$} & \multirow{2}{*}{ Feeding behaviour } & \multirow{2}{*}{$\begin{array}{c}\text { Commercial } \\
\text { value }\end{array}$} & \multirow{2}{*}{$\begin{array}{l}\text { IUCN } \\
\text { status }\end{array}$} & \multicolumn{2}{|c|}{ Ametlla } & \multicolumn{2}{|c|}{ Mataró } & \multicolumn{2}{|c|}{ Medes Islands } & \multicolumn{2}{|c|}{ Palamós } \\
\hline & & & & & Mean & Std & Mean & Std & Mean & Std & Mean & Std \\
\hline Ammodytidae & & & & & & & & & & & & \\
\hline $\begin{array}{l}\text { Gymnammomytes cicerelus* } \\
\text { Apogonidae }\end{array}$ & $\geq 2.8$ & Filtering plankton & Unknown & $\mathrm{LC}$ & 0.06 & 0.08 & 0.20 & 0.36 & 0.00 & 0.01 & 0.01 & 0.02 \\
\hline $\begin{array}{l}\text { Apogon imberbis } \\
\text { Atherinidae }\end{array}$ & $\geq 2.8$ & Hunting macrofauna & NONE & $\mathrm{LC}$ & 0.47 & 0.34 & 0.54 & 0.28 & 0.41 & 0.38 & 0.68 & 0.17 \\
\hline Atherina sp. & $\geq 2.8$ & Hunting macrofauna & Low & $\mathrm{LC}$ & 0.54 & 0.25 & & & 0.41 & 0.26 & 0.68 & 0.30 \\
\hline Blenniidae & & & & & 0.47 & & 0.46 & & 0.33 & & 0.37 & \\
\hline Aidablennius sphynx (M) & $2.2-2.79$ & Grazing & NONE & $\mathrm{LC}$ & 0.31 & 0.27 & & & 0.48 & 0.33 & 0.64 & 0.31 \\
\hline Coryphoblennius galerita & $2-2.19$ & Grazing & None & $\mathrm{LC}$ & 0.03 & 0.06 & & & 0.05 & 0.06 & 0.11 & 0.15 \\
\hline Lipophrys trigloides & $2.2-2.79$ & Variable & NONE & $\mathrm{LC}$ & 0.46 & 0.17 & & & 0.26 & 0.30 & 0.29 & 0.27 \\
\hline Microlipophrys canevae & $2-2.19$ & Variable & NONE & $\mathrm{LC}$ & 0.76 & 0.17 & & & 0.53 & 0.32 & 0.32 & 0.30 \\
\hline Microlipophrys dalmatinus* & $2.2-2.79$ & Variable & NONE & $\mathrm{LC}$ & 0.25 & 0.23 & & & 0.12 & 0.22 & 0.05 & 0.06 \\
\hline Microlipophrys nigriceps & $2.2-2.79$ & Variable & NONE & $\mathrm{LC}$ & 0.29 & 0.17 & 0.15 & 0.18 & 0.19 & 0.22 & 0.02 & 0.06 \\
\hline Parablennius gattorugine & $2.2-2.79$ & Grazing & Unknown & $\mathrm{LC}$ & 0.8 & 0.1 & 0.45 & 0.17 & 0.47 & 0.25 & 0.79 & 0.14 \\
\hline Parablennius incognitus & $2.2-2.79$ & Variable & NONE & $\mathrm{LC}$ & 0.98 & 0.21 & & & 0.45 & 0.36 & 0.49 & 0.23 \\
\hline Parablennius pilicornis & $\geq 2.8$ & NA & NONE & $\mathrm{LC}$ & 0.89 & 0.13 & 0.77 & 0.18 & 0.80 & 0.21 & 0.88 & 0.19 \\
\hline Parablennius rouxi & $2.2-2.79$ & Variable & NONE & $\mathrm{LC}$ & 0.04 & 0.06 & 0.49 & 0.3 & 0.17 & 0.11 & 0.09 & 0.09 \\
\hline Parablennius sanguinolentus & $2-2.19$ & Grazing & Unknown & $\mathrm{LC}$ & 0.54 & 0.24 & & & 0.32 & 0.36 & 0.65 & 0.30 \\
\hline Parablennius tentacularis & $\geq 2.8$ & Browsing & NONE & $\mathrm{LC}$ & & & & & 0.02 & 0.04 & 0.05 & 0.09 \\
\hline Parablennius zvonimiri & $2.2-2.79$ & Variable & NONE & $\mathrm{LC}$ & 0.62 & 0.13 & 0.44 & 0.21 & 0.44 & 0.24 & 0.46 & 0.28 \\
\hline Scartella cristata & $2-2.19$ & Grazing & NONE & $\mathrm{LC}$ & 0.11 & 0.14 & & & & & & \\
\hline Bothidae & & & & & & & & & & & & \\
\hline $\begin{array}{l}\text { Bothus podas } \\
\text { Callionymidae }\end{array}$ & $\geq 2.8$ & Predator & High & $\mathrm{LC}$ & 0.38 & 0.26 & 0.08 & 0.1 & 0.04 & 0.09 & 0.02 & 0.06 \\
\hline $\begin{array}{l}\text { Callionymus pusillus* } \\
\text { Carangidae }\end{array}$ & $\geq 2.8$ & Predator & NONE & $\mathrm{LC}$ & 0.01 & 0.02 & & & 0.05 & 0.07 & & \\
\hline $\begin{array}{l}\text { Trachurus sp. } \\
\text { Centracanthidae }\end{array}$ & $\geq 2.8$ & Predator & Medium & VU & 0.02 & 0.03 & 0.08 & 0.13 & 0.04 & 0.07 & 0.02 & 0.06 \\
\hline $\begin{array}{l}\text { Spicara smaris } \\
\text { Clinidae }\end{array}$ & $\geq 2.8$ & Predator & Low & $\mathrm{LC}$ & & & & & 0.02 & 0.05 & 0.02 & 0.04 \\
\hline Clinitrachus argentatus & $\geq 2.8$ & Predator & NONE & $\mathrm{LC}$ & 0.09 & 0.11 & & & 0.02 & 0.07 & 0.06 & 0.13 \\
\hline Con & & & & & & & & & & & & \\
\hline Conger conger $(\mathrm{P})$ & $\geq 2.8$ & Predator & Medium & $\mathrm{LC}$ & 0.19 & 0.18 & 0.33 & 0.18 & 0.43 & 0.24 & 0.14 & 0.14 \\
\hline Gadidae & & & & & & & & & & & & \\
\hline Phycis phycis (A,M,P) & $\geq 2.8$ & Predator & High & $\mathrm{LC}$ & 0.03 & 0.06 & 0.09 & 0.22 & 0.42 & 0.14 & 0.04 & 0.08 \\
\hline Gobiesocidae & & & & & & & & & & & & \\
\hline Lepadogaster candollei & $2.2-2.79$ & NA & NONE & N.E. & & & 0.20 & 0.4 & 0.26 & 0.22 & 0.34 & 0.27 \\
\hline Lepadogaster lepadogaster & NA & NA & NONE & $\mathrm{LC}$ & 0.18 & 0.18 & & & 0.15 & 0.14 & 0.33 & 0.32 \\
\hline Gobiidae & & & & & 0.40 & & 0.23 & & 0.20 & & 0.39 & \\
\hline Gobius bucchichi & $2.2-2.79$ & Variable & NONE & $\mathrm{LC}$ & 0.97 & 0.11 & 0.65 & 0.31 & 0.78 & 0.17 & 0.94 & 0.19 \\
\hline Gobius cobitis & $2.2-2.79$ & Variable & NONE & N.E. & 0.43 & 0.35 & & & 0.23 & 0.24 & 0.52 & 0.21 \\
\hline Gobius cruentatus & $\geq 2.8$ & Browsing & NONE & $\mathrm{LC}$ & 0.41 & 0.3 & 0.05 & 0.12 & 0.06 & 0.18 & 0.35 & 0.26 \\
\hline Gobius geniporus & $\geq 2.8$ & NA & NONE & $\mathrm{LC}$ & 0.24 & 0.18 & 0.80 & 0.16 & 0.29 & 0.14 & 0.49 & 0.24 \\
\hline Gobius niger & $\geq 2.8$ & plankton feeding & NONE & $\mathrm{LC}$ & & & 0.01 & 0.03 & 0.01 & 0.02 & 0.09 & 0.11 \\
\hline Gobius paganellus & $\geq 2.8$ & Predator & NONE & $\mathrm{LC}$ & 0.6 & 0.05 & 0.03 & 0.05 & 0.34 & 0.31 & 0.65 & 0.31 \\
\hline Gobius vittatus & $2.2-2.79$ & Variable & NONE & $\mathrm{LC}$ & & & 0.01 & 0.03 & 0.03 & 0.06 & & \\
\hline Pomatoschistus $\mathrm{sp}^{*}$ & 3.2 & Hunting macrofauna & NONE & $\mathrm{LC}$ & 0.55 & 0.34 & 0.16 & 0.24 & 0.11 & 0.19 & 0.13 & 0.14 \\
\hline Gobius xanthocephalus & NA & NA & NONE & $\mathrm{LC}$ & 0.12 & 0.16 & 0.03 & 0.05 & 0.09 & 0.11 & 0.03 & 0.07 \\
\hline Zebrus zebrus* & NA & NA & NONE & $\mathrm{LC}$ & 0.06 & 0.06 & & & 0.01 & 0.03 & 0.03 & 0.10 \\
\hline Haemulidae & & & & & & & & & & & & \\
\hline Pomadasys incisus* & $\geq 2.8$ & Predator & Medium & $\mathrm{LC}$ & 0.13 & 0.19 & & & 0.01 & 0.02 & 0.03 & 0.07 \\
\hline Labridae & & & & & 0.55 & & 0.57 & & 0.57 & & 0.56 & \\
\hline Coris julis & $\geq 2.8$ & Predator & Low & $\mathrm{LC}$ & 0.99 & 0.03 & 0.99 & 0.03 & 0.98 & 0.04 & 0.99 & 0.04 \\
\hline Ctenolabrus rupestris & $\geq 2.8$ & Predator & Low & $\mathrm{LC}$ & 0.12 & 0.19 & 0.76 & 0.14 & 0.25 & 0.19 & 0.66 & 0.11 \\
\hline Labrus merula (A,M,P) & $\geq 2.8$ & Predator & Low & $\mathrm{LC}$ & 0.68 & 0.26 & 0.57 & 0.17 & 0.99 & 0.08 & 0.74 & 0.14 \\
\hline Labrus mixtus & $\geq 2.8$ & Predator & Low & $\mathrm{LC}$ & & & & & 0.06 & 0.09 & 0.02 & 0.06 \\
\hline Labrus viridis (A,M,P) & $\geq 2.8$ & Predator & NONE & VU & 0.11 & 0.11 & 0.03 & 0.06 & 0.71 & 0.20 & 0.37 & 0.16 \\
\hline Symphodus cinereus & $\geq 2.8$ & Predator & Low & $\mathrm{LC}$ & 0.82 & 0.22 & 0.49 & 0.3 & 0.38 & 0.31 & 0.18 & 0.16 \\
\hline Symphodus doderleini & $\geq 2.8$ & Predator & NONE & $\mathrm{LC}$ & 0.06 & 0.11 & 0.03 & 0.06 & 0.09 & 0.07 & 0.19 & 0.08 \\
\hline Symphodus mediterraneus & $\geq 2.8$ & Predator & Low & $\mathrm{LC}$ & 0.3 & 0.13 & 0.81 & 0.09 & 0.72 & 0.19 & 0.77 & 0.17 \\
\hline Symphodus melanocercus & $\geq 2.8$ & Predator & NONE & $\mathrm{LC}$ & 0.04 & 0.06 & 0.33 & 0.19 & 0.62 & 0.27 & 0.59 & 0.21 \\
\hline Symphodus melops & $\geq 2.8$ & Predator & Low & $\mathrm{LC}$ & 0.32 & 0.19 & 0.01 & 0.02 & 0.10 & 0.09 & 0.10 & 0.16 \\
\hline Symphodus ocellatus & $\geq 2.8$ & Variable & NONE & $\mathrm{LC}$ & 0.98 & 0.06 & 0.69 & 0.2 & 0.62 & 0.26 & 0.84 & 0.15 \\
\hline Symphodus roissali & $\geq 2.8$ & Predator & Low & $\mathrm{LC}$ & 0.96 & 0.04 & 0.97 & 0.1 & 0.97 & 0.15 & 0.99 & 0.12 \\
\hline Symphodus rostratus & $\geq 2.8$ & Predator & NONE & $\mathrm{LC}$ & 0.66 & 0.14 & 0.29 & 0.11 & 0.58 & 0.20 & 0.37 & 0.16 \\
\hline Symphodus tinca & $\geq 2.8$ & Predator & Low & $\mathrm{LC}$ & 0.94 & 0.07 & 0.97 & 0.06 & 0.99 & 0.07 & 0.93 & 0.08 \\
\hline Thalassoma pavo & $\geq 2.8$ & Predator & Low & $\mathrm{LC}$ & 0.72 & 0.42 & 0.97 & 0.05 & 0.50 & 0.29 & 0.73 & 0.34 \\
\hline Moronidae & & & & & & & & & & & & \\
\hline Dicentrarchus labrax (M,P) & $\geq 2.8$ & Predator & High & $\mathrm{LC}$ & 0.47 & 0.22 & & & 0.44 & 0.27 & 0.08 & 0.16 \\
\hline Dicentrarchus punctatus* & $\geq 2.8$ & Predator & Low & $\mathrm{LC}$ & 0.94 & 0.1 & 0.75 & 0.16 & 0.65 & 0.29 & 0.42 & 0.42 \\
\hline & $2-2.19$ & Variable & Low & $\mathrm{LC}$ & 0.79 & 0.14 & 0.02 & 0.04 & 0.85 & 0.14 & 0.81 & 0.21 \\
\hline
\end{tabular}


Table 2 (cont.). - Fish species average catch image rate and standard deviation by location. The letters within brackets next to the species correspond to those with a catch image rate significantly higher in the Medes Marine Reserve; each letter corresponds to the location where the significant differences were observed. Asterisks indicate a species described for the first time in the Reserve.

\begin{tabular}{|c|c|c|c|c|c|c|c|c|c|c|c|c|}
\hline \multirow{2}{*}{ Species } & \multirow{2}{*}{$\begin{array}{c}\text { Feeding } \\
\text { (trophic } \\
\text { levels) }\end{array}$} & \multirow{2}{*}{ Feeding behaviour } & \multirow{2}{*}{$\begin{array}{c}\text { Commercial } \\
\text { value }\end{array}$} & \multirow{2}{*}{$\begin{array}{l}\text { IUCN } \\
\text { status }\end{array}$} & \multicolumn{2}{|c|}{ Ametlla } & \multicolumn{2}{|c|}{ Mataró } & \multicolumn{2}{|c|}{ Medes Islands } & \multicolumn{2}{|c|}{ Palamós } \\
\hline & & & & & Mean & Std & Mean & Std & Mean & Std & Mean & Std \\
\hline \multicolumn{13}{|l|}{ Mullidae } \\
\hline Mullus surmuletus & $\geq 2.8$ & Predator & High & $\mathrm{LC}$ & 0.65 & 0.22 & 0.96 & 0.06 & 0.89 & 0.11 & 0.76 & 0.27 \\
\hline \multicolumn{13}{|l|}{ Muraenidae } \\
\hline Muraena helena & $\geq 2.8$ & Predator & Low & $\mathrm{LC}$ & 0.3 & 0.77 & 0.28 & 0.17 & 0.36 & 0.17 & 0.20 & 0.09 \\
\hline \multicolumn{13}{|l|}{ Pomacentridae } \\
\hline Chromis chromis & $\geq 2.8$ & Predator & Unknown & $\mathrm{LC}$ & 0.95 & 0.07 & 0.96 & 0.06 & 0.94 & 0.03 & 0.98 & 0.07 \\
\hline \multicolumn{13}{|l|}{ Sciaenidae } \\
\hline Sciaena umbra $(\mathrm{M}, \mathrm{P})$ & $\geq 2.8$ & Predator & High & NT & 0.3 & 0.28 & 0.03 & 0.04 & 0.29 & 0.22 & & \\
\hline \multicolumn{13}{|l|}{ Scorpaenidae } \\
\hline Scorpaena maderensis & $\geq 2.8$ & Predator & Low & $\mathrm{LC}$ & 0.09 & 0.13 & 0.41 & 0.19 & 0.03 & 0.04 & 0.09 & 0.11 \\
\hline Scorpaena notata & $\geq 2.8$ & Predator & Low & $\mathrm{LC}$ & 0.47 & 0.22 & 0.90 & 0.09 & 0.21 & 0.19 & 0.51 & 0.21 \\
\hline Scorpaena porcus & $\geq 2.8$ & Predator & Low & $\mathrm{LC}$ & 0.82 & 0.12 & 0.44 & 0.22 & 0.24 & 0.28 & 0.58 & 0.19 \\
\hline Scorpaena scrofa $(\mathrm{P})$ & $\geq 2.8$ & Predator & High & $\mathrm{LC}$ & 0.3 & 0.32 & 0.63 & 0.26 & 0.61 & 0.33 & 0.03 & 0.04 \\
\hline \multicolumn{13}{|l|}{ Serranidae } \\
\hline Epinephelus marginatus (A,M,P) & $\geq 2.8$ & Predator & High & EN & 0.21 & 0.17 & 0.15 & 0.17 & 0.49 & 0.16 & 0.08 & 0.07 \\
\hline Serranus cabrilla & $\geq 2.8$ & Predator & Medium & $\mathrm{LC}$ & 0.82 & 0.37 & 0.98 & 0.03 & 0.97 & 0.05 & 0.96 & 0.05 \\
\hline Serranus scriba & $\geq 2.8$ & Predator & Medium & $\mathrm{LC}$ & 0.91 & 0.17 & 0.97 & 0.05 & 0.94 & 0.05 & 0.76 & 0.26 \\
\hline Sparidae & & & & & 0.43 & & 0.46 & & 0.45 & & 0.31 & \\
\hline Boops boops & $\geq 2.8$ & plankton feeding & Low & $\mathrm{LC}$ & 0.31 & 0.22 & 0.77 & 0.22 & 0.22 & 0.26 & 0.32 & 0.33 \\
\hline Dentex dentex & $\geq 2.8$ & Predator & High & VU & 0.08 & 0.07 & 0.06 & 0.06 & 0.16 & 0.24 & 0.04 & 0.11 \\
\hline Diplodus annularis & $\geq 2.8$ & Predator & Low & LC & 0.58 & 0.26 & 0.75 & 0.14 & 0.30 & 0.39 & 0.15 & 0.16 \\
\hline Diplodus cervinus (A) & $\geq 2.8$ & Predator & Low & $\mathrm{LC}$ & 0.06 & 0.09 & 0.21 & 0.25 & 0.42 & 0.20 & 0.14 & 0.21 \\
\hline Diplodus puntazzo & $\geq 2.8$ & Predator & Low & $\mathrm{LC}$ & 0.94 & 0.1 & 0.75 & 0.16 & 0.65 & 0.29 & 0.42 & 0.42 \\
\hline Diplodus sargus $(\mathrm{P})$ & $\geq 2.8$ & Predator & High & $\mathrm{LC}$ & 0.96 & 0.04 & 0.85 & 0.11 & 0.98 & 0.04 & 0.77 & 0.14 \\
\hline Diplodus vulgaris & $\geq 2.8$ & Predator & Low & $\mathrm{LC}$ & 1.00 & 0 & 0.98 & 0.04 & 0.95 & 0.05 & 0.90 & 0.12 \\
\hline Lithognathus mormyrus & $\geq 2.8$ & Predator & Medium & $\mathrm{LC}$ & 0.13 & 0.11 & & & 0.17 & 0.28 & 0.03 & 0.05 \\
\hline Oblada melanura & $\geq 2.8$ & Predator & Low & LC. & 0.96 & 0.04 & 0.83 & 0.1 & 0.82 & 0.14 & 0.66 & 0.22 \\
\hline Pagellus acarne & $\geq 2.8$ & Predator & Medium & $\mathrm{LC}$ & 0.1 & 0.17 & 0.01 & 0.03 & 0.10 & 0.12 & 0.06 & 0.11 \\
\hline Pagellus erythrinus & $\geq 2.8$ & Predator & Medium & $\mathrm{LC}$ & 0.01 & 0.02 & 0.01 & 0.03 & 0.05 & 0.11 & 0.02 & 0.04 \\
\hline Pagrus pagrus & $\geq 2.8$ & Predator & High & $\mathrm{LC}$ & 0.11 & 0.2 & 0.01 & 0.03 & & & 0.08 & 0.10 \\
\hline Sarpa salpa & $2-2.19$ & Grazing & Low & $\mathrm{LC}$ & 0.98 & 0.13 & 0.70 & 0.18 & 0.96 & 0.05 & 0.96 & 0.08 \\
\hline Spicara smaris & $\geq 2.8$ & Predator & Low & $\mathrm{LC}$ & & & & & 0.02 & 0.05 & 0.02 & 0.04 \\
\hline Spicara maena & $\geq 2.8$ & plankton feeding & Low & $\mathrm{LC}$ & 0.03 & 0.06 & 0.18 & 0.14 & 0.29 & 0.25 & 0.08 & 0.08 \\
\hline Spondyliosoma cantharus & $\geq 2.8$ & Variable & High & $\mathrm{LC}$ & 0.25 & 0.22 & 0.53 & 0.26 & 0.24 & 0.22 & 0.03 & 0.04 \\
\hline \multicolumn{13}{|l|}{ Sphyraenidae } \\
\hline Sphyraena sp. & $\geq 2.8$ & Predator & Medium & $\mathrm{LC}$ & 0.01 & 0.02 & & & 0.01 & 0.02 & 0.04 & 0.10 \\
\hline \multicolumn{13}{|l|}{ Torpedinidae } \\
\hline Torpedo marmorata & $\geq 2.8$ & Predator & NONE & DD & 0.06 & 0.13 & 0.05 & 0.07 & 0.06 & 0.11 & & \\
\hline \multicolumn{13}{|l|}{ Trachinidae } \\
\hline Trachinus sp. & $\geq 2.8$ & Predator & Low & $\mathrm{LC}$ & 0.02 & 0.03 & 0.08 & 0.14 & 0.04 & 0.07 & 0.02 & 0.06 \\
\hline \multicolumn{13}{|l|}{ Tripterygiidae } \\
\hline Tripterygion delaisi & $\geq 2.8$ & Predator & NONE & $\mathrm{LC}$ & 0.5 & 0.31 & 1.00 & 0.15 & 0.81 & 0.16 & 0.85 & 0.08 \\
\hline Tripterygion melanurus & $\geq 2.8$ & Predator & NONE & $\mathrm{LC}$ & 0.14 & 0.32 & 0.14 & 0.38 & 0.10 & 0.30 & 0.11 & 0.32 \\
\hline Tripterygion tripteronotus & $\geq 2.8$ & Predator & NONE & $\mathrm{LC}$ & 0.46 & 0.37 & 0.11 & 0.06 & 0.66 & 0.23 & 0.95 & 0.12 \\
\hline
\end{tabular}

the species identified until now as Gobius bucchichi in this region, should be considered to be the recently described G. incognitus (Kovačić and Šanda 2016). The lists of species observed at the Medes Islands are 7 species that do not appear in the previous available fish inventory in this MPA (Dufour et al. 2007). Species richness and Margalef diversity indices showed significant differences among locations (Fig. 2). The multiple comparison Tukey test showed that only Mataró has a significantly lower species richness (Table 1), mainly due to the lower number of species of blenniids and gobids (Table 2).

Cluster analysis based on the species catch rates by location and year yielded four distinctive clusters (Fig. 3 ) corresponding to each specific location. Regardless of the year, each location was grouped in the same cluster, with one exception, the Medes Islands in 2015. The silhouette analysis (mean silhouette width $=0.46$ ) revealed that all contests had positive silhouette widths, which is indicative of correct classification within groups. The two-dimensional ordination of the
29 contests (Fig. 4) yielded a moderate level of ordination (stress $=0.14$ ), reflecting cluster separation through a gradual continuum of change. A gradient from north to south was observed among locations near the shoreline (Medes, Palamós and L'Ametlla). Consistently, the results of the cluster analysis showed that the most dissimilar group was represented by the contests held in Mataró.

The first cluster, grouping all the samples from Mataró, had a low number of gobiid and bleniid species, scarcity of mugilids and the absence of Atherinidae and European seabass (Dicentrarchus labrax), which mainly marked the difference of this group. An additional difference was the high values of species such as Scorpaena notata, Thalassoma pavo, Boops boops and Gobius geniporus.

The second cluster grouped all the contests of L'Ametlla de Mar, the southernmost location (the last contest held at the Medes Islands was also part of this group). The main differences compared with other groups were the high abundance of the genera Pagel- 


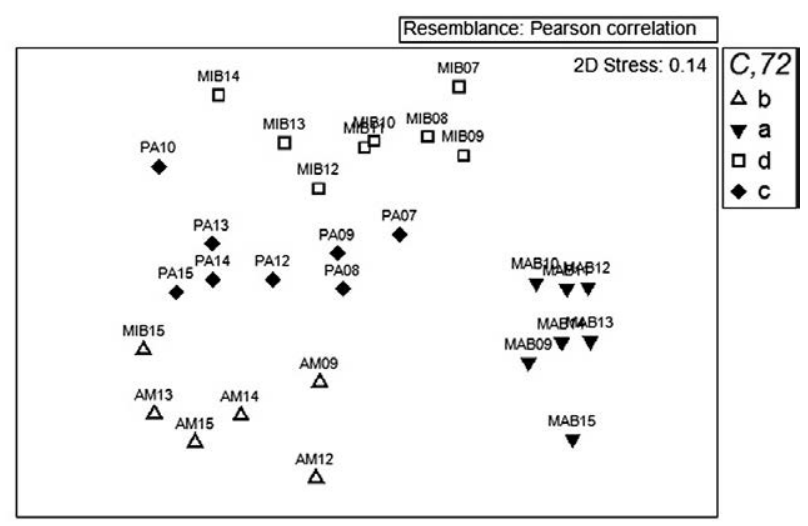

Fig. 4. - 2-d nMDS ordination plot for the 29 contests. Superimposed symbols for the different clusters.

lus, Symphodus cinereus and Parablennius incognitus together with the scarcity of Ctenolabrus rupestris, $M i$ crolipophrys canevae, Symphodus melanocercus and Diplodus cervinus. In this group the relative abundance of European seabass was also high, and only comparable with the values observed at the Medes Islands.

The third and fourth cluster comprised the contests held at the northernmost location corresponding to a non-protected and protected area respectively with the exception of the most recent year (see above) for the latter group. The main features of this group were the low relative abundance of gobiids, the maximum observed abundance of some species of labrids (Symphodus melanocercus, Symphodus viridis and Labrus merula) and the scarcity of S. ocellatus. This group was also characterized by high abundance of commercial species (e.g. Epinephelus marginatus, Conger conger and Phycis phycis), including the red scorpion fish $(S$. scrofa), but it also had the lowest abundance of the other three species of the same genus $(S$. notata, $S$. porcus and $S$. maderensis).

Although the clusters did not show any temporal variability within locations, except for the last contest held in Medes Islands, some shifts can be depicted. Some temporal changes were observed in the contest distances within the Medes Islands cluster, in which the 2013 and 2014 contests were clearly separated from the earlier ones. Similarly, differences were observed within the Palamós (cluster C) contests: those from 2012 onwards were distant from the previous ones, while in L'Ametlla the temporal pattern was more gradual.

The post hoc test showed significantly higher catch rates at the Medes Islands MPA with respect to the other locations (Table 2). These higher rates were mostly observed in species of commercial interest such as $P$. phycis and E. marginatus, but the list can be extended to Conger conger, D. labrax, S. umbra, S. scrofa, D. cervinus and D. sargus. In addition, the MPA also showed significantly higher catch rates for two species of labrids that are of negligible commercial interest but are highly targeted by spear fishers (L. merula and $L$. viridis). Furthermore, the relative abundance of $\mathrm{S}$. $\mathrm{ci}$ nereus decreased from southern to northern locations, while $S$. melanocercus displayed the opposite pattern.

\section{DISCUSSION}

Overall, the obtained outcomes show that freediving fish photography contests could be a very useful tool for analysing fish assemblages and species richness in the littoral system. In general, fish classification results grouped each location separately, as expected from their differences in latitude, depth range and habitat. In addition, the already known effect of the Medes Islands MPA (García-Rubies and Zabala 1990, García-Rubies et al. 2013) was evidenced by the higher abundance of species of high commercial values.

The high number of species recorded in this study exceeds by far those reported in any published study on littoral communities carried out in the Mediterranean (e.g. Consoli et al. 2016), and is only comparable to but still higher than the number of species reported in the marine reserve of Ustica Island (La Mesa and Vacchi 1999). It is worth mentioning that the high number of species reported in the study of Ustica Island could be due to the combined sampling methodology used (strip transects by SCUBA, SCUBA diving tracts and snorkelling), which minimized the constraints inherent in survey methods, particularly for cryptic species (Brock 1982, Willis 2001, MacNeil et al. 2008). This indicates the effectiveness of the analysed information source for measuring fish species richness with a single data source.

In the Mediterranean, the littoral fish community is under various anthropogenic threats (Bianchi and Morri 2000, Claudet and Fraschetti 2010, Costello et al. 2010), but systematic monitoring is limited to a small number of commercial species or to protected areas (e.g. Coll et al. 2012, García-Rubies et al. 2013). Although overall littoral fish monitoring would be unrealistic to finance, many commercial groups, such as sparids, are not subject to assessment, so they are not under any systematic monitoring programmes, and this lack of information is hard to justify. The species recorded in this study that have a known status are mainly on the least-concern list: only one is endangered (E. marginatus) and one is considered vulnerable (L. viridis).

Among the results shown in this study, of particular relevance is the capacity of free-diving photography contests to count and assess cryptic species, which can be missed by underwater visual censuses (Ackerman and Bellwood 2000, Willis 2001, Smith-Vaniz et al. 2006). A total number of 32 cryptic species were photographed during these contests, a figure that is also higher than in any previous studies targeting this group based either on visual censuses (e.g. Macpherson 1994, La Mesa et al. 2006, Bussotti et al. 2015) or destructive techniques (Patzner 1999, Kovačić et al. 2012, Glavičić et al. 2016). Although they contribute greatly to the species richness of littoral systems, small cryptic species are perceived as minnows (small non-game or non-commercial fish species) due to their small size and cryptic behaviour. Consequently, they are generally overlooked or underestimated (Ackerman and Bellwood 2000, Willis 2001), and it is difficult to gain support for monitoring and conserving them (Sheldon 
1988). Therefore, an important part of the strictly substratum-dependent littoral fish community is usually ignored, preventing any changes from being detected both in the community and in the microhabitat of the substratum.

Overall, the most abundant species were $C$. julis, $S$. roissali, $S$. tinca, $S$. cabrilla, $D$. vulgaris and $C$. chromis. In numerous studies these species have been reported as the most common in the rocky reef habitats of the Mediterranean littoral system (Bell 1983, García-Rubies and Zabala 1990, Claudet et al. 2006). However, the most abundant species differed between locations. The most abundant species in Mataró was T. delaissi. The preferred habitat of this species, flat, rocky and sheltered from wave action of the open sea (La Mesa et al. 2004), match the Mataró habitat location, a small, relatively flat, natural rock over $150 \mathrm{~m}$ in length. Further characteristics of this location, offshore at a minimum depth of $6 \mathrm{~m}$ that minimizes wave action and light, are also considered to be preferred by $T$. delaisi (Zander and Heymer 1970). Mataró's minimum depth also explained the absence of species typical of shallow waters, such as many species of blenniids (e.g. A. sphynx, L. trigloides and M. canevae), whose highest diversity (Kotrschal 1988, Macpherson 1994) is restricted to the narrow depth zone from 0 to $1 \mathrm{~m}$ (Illich and Kotrschal 1990) and the negative correlation between depth and blenniid diversity was recently proved (Tiralongo et al. 2016). This absence was also detected for some other species characteristic of shallow waters (C. argentatus and Z. zebrus). Another interesting observation in Mataró was the coexistence with high relative abundances of the four species of scorpenids ( $S$. maderensis, S. notata, S. porcus and S. scrofa). On this basis, we could reject the mutual species displacements by interspecific competition for habitat or food resources that have been hypothesized for this group (La Mesa et al. 2004). However, based on the findings in the MPA, we cannot entirely reject this hypothesis. In the MPA, while $S$. scrofa was highly abundant, the other species of scorpaenids showed values generally below those observed in the non-protected locations. $S$. scrofa is bigger than the other species (Petrakis and Stergiou 1995, Ordines and Massuti 2009, La Mesa et al. 2010) and under the protection effect the species can get older and bigger (e.g. Halpern and Warner 2003) and easier to see.

The results on species richness and diversity index showed no positive effects on the MPA, as has been proposed in other studies (Guilhaumon et al. 2015). The results are indicative of the positive effect of the MPA on species of commercial interest, but not on all species, because responses to protection can be highly variable among fish taxa (Claudet et al. 2006). The positive effect of the MPA in contrast with the abundance observed in the closest comparable nonprotected location (Palamós) was unquestionable for species of high commercial value. A total of eight species showed higher and significant values within the MPA: C. conger, P. phycis, D. labrax, S. umbra, $S$. scrofa, E. marginatus and $D$. sargus. The recovery of the dusky grouper in MPAs has been reported in numerous studies (e.g. Bell 1983, García-Rubies and Zabala 1990, Sahyoun et al. 2013) as has that of white seabream (Sahyoun et al. 2013). The positive effect in this particular MPA, the Medes Islands, has already been observed for most of the above-mentioned species (i.e. D. labrax, E. marginatus, D. cervinus, $S$. aurata and $S$. umbra) (García-Rubies et al. 2013). In addition, two species of labrids (L. merula and $L$. viridis) of no commercial interest but of recreational spear fishing interest were also favoured by the protection. L. viridis is considered vulnerable and its protection is recommended. The lack of information on fish sizes prevents us from analysing the well-known positive effect of MPA on this key indicator (Lester et al. 2009).

A different argument is that the response to protection can also be negative for certain taxa, and changes in fish assemblages in MPAs could occur because predation pressure is expected to be higher (Francour 1994, Ashworth and Ormond 2005). A potential negative effect on small cryptic species has been suggested (Willis and Anderson 2003, Claudet et al. 2006), but our results confirm that only two species were significantly higher at the unprotected site ( $P$. gattorugine and $T$. tripteronotus). However, not all the differences in species abundance can be attributed to protection measures or to the type of data, because we also found a latitudinal gradient of variability that was unrelated to these factors. $S$. melanocercus, which prefers rocky areas and seagrass beds, decreases from north to south, while $S$. cinereus and S. ocellatus, which generally inhabits seagrass beds and sometimes soft bottoms and estuarine lagoons, increases from north to south. This partitioning is consistent with the previous categorization in the Catalan littoral system based on shore-based fishing tournament reports (Gordoa 2009, Boada et al 2017).

The type of information used in this study has several weaknesses, such as a potential underestimation of the most frequent species because the number of photographs per species and participant is limited to one, and the possibility that the same fish could be photographed by different participants. However, despite the potential weaknesses of photographic free-diving contest data, the results of this study show their effectiveness for evaluating species richness, in particular of cryptobenthic species, and for analysing littoral fish communities. Furthermore, it has also proved its worth as a tool for updating fish inventories. We can summarize that this type of contest enhances competition between participants to obtain maximum fish diversity. Additional positive aspects of this information source are that it is cost-effective, non-destructive, a potential observatory, an platform for interaction between scientists and free divers, and an alternative for spear fishers in MPAs. We conclude that the monitoring of photographic free-diving contests could be a complementary information source to scientific monitoring.

\section{ACKNOWLEDGEMENTS}

The authors are very grateful to the Catalan Federation of Underwater Activities (FECDAS) for provid- 
ing all the information analysed in this study and in particular to Carles Font, delegate of apnoea photohunting and responsible for compiling the database of these contests and to Catherine Stonehouse for revising and improving the original English manuscript. We also thank the anonymous referees for their useful comments.

\section{REFERENCES}

Ackerman J.L., Bellwood D.R. 2000. Reef fish assemblages: a reevaluation using enclosed rotenone stations. Mar. Ecol. Prog. Ser. 206: 227-237. https://doi.org/10.3354/meps206227

Ashworth J., Ormond R. 2005. Effects of fishing pressure and trophic group on abundance and spillover across boundaries of a no-take zone. Biol. Conserv. 12: 333-344. https://doi.org/10.1016/j.biocon.2004.05.006

Azzurro E., Moschella P., Maynou F. 2011. Tracking Signals of Change in Mediterranean Fish Diversity Based on Local Ecological Knowledge. PLoS ONE 6: e24885. https://doi.org/10.1371/journal.pone.0024885

Bell J.D. 1983. Effects of depth and marine reserve fishing restrictions on the structure of a rocky reef fish assemblage in the north-western Mediterranean Sea. J. Appl. Ecol. 20: 357-369. https://doi.org/10.2307/2403513

Benedetti-Cecchi L., Pannacciulli F., Bulleri F., et al. 2001. Predicting the consequences of anthropogenic disturbance: large-scale effects of loss of canopy algae on rocky shores. Mar. Ecol. Prog. Ser. 214: 137-150. https://doi.org/10.3354/meps214137

Bianchi C.N., Morri C. 2000. Marine biodiversity of the Mediterranean Sea: situation, problems and prospects for future research. Mar. Pollut. Bull. 40: 367-376. https://doi.org/10.1016/S0025-326X(00)00027-8

Boada J., Sagué O., Gordoa A. 2017. Spearfishing data reveals the littoral fish communities' association to coastal configuration. Estuar. Coast. Shelf Sci. 199: 152-160. https://doi.org/10.1016/j.ecss.2017.10.007

Boudouresque C., Cadiou G., Le Diréac'h L. 2005. Marine protected areas: a tool for coastal areas management. Nato Science Series IV: Earth and Environmental Sciences. Springer Netherlands, pp. 29-52.

Brock R.E. 1982. A critique of the visual census method for assessing coral reef fish populations. Bull. Mar. Sci. 32: 269-276.

Bussotti S., Di Franco A., Francour P., et al. 2015. Fish assemblages of Mediterranean marine caves. PloS ONE 10: e0122632. https://doi.org/10.1371/journal.pone.0122632

Cappo M., Speare P., De'ath G. 2004. Comparison of baited remote underwater video stations (BRUVS) and prawn (shrimp) trawls for assessments of fish biodiversity in inter-reefal areas of the Great Barrier Reef Marine Park. J. Exp. Mar. Biol. Ecol. 302: 123-152. https://doi.org/10.1016/j.jembe.2003.10.006

Claudet J., Fraschetti S. 2010. Human-driven impacts on marine habitats: a regional meta-analysis in the Mediterranean Sea. Biol. Conserv. 143: 2195-2206. https://doi.org/10.1016/j.biocon.2010.06.004

Claudet J., Pelletier D., Jouvenel J.Y., et al. 2006. Assessing the effects of marine protected area (MPA) on a reef fish assemblage in a northwestern Mediterranean marine reserve: Identifying community-based indicators. Biol. Conserv. 130: 349-369. https://doi.org/10.1016/j.biocon.2005.12.030

Coll J., Linde M., García-Rubies A., et al. 2004. Spear fishing in the Balearic Islands (west central Mediterranean): species affected and catch evolution during the period 1975-2001. Fish. Res. 70: 97-111. https://doi.org/10.1016/j.fishres.2004.05.004

Coll J., Garcia-Rubies A., Morey G., et al. 2012. The carrying capacity and the effects of protection level in three marine protected areas in the Balearic Islands (NW Mediterranean). Sci. Mar. 76: 809-826.

Connell S.D., Samoilys M.A., Lincoln Smith M.P., et al. 1998 Comparisons of abundance of coral-reef fish: Catch and effort surveys vs visual census. Aust. J. Ecol. 23: 579-586. https://doi.org/10.1111/j.1442-9993.1998.tb00768 x

Consoli P., Esposito V., Battaglia P., et al. 2016. Fish Distribution and Habitat Complexity on Banks of the Strait of Sicily (Central Mediterranean Sea) from Remotely-Operated Vehicle (ROV) Explorations. PloS ONE 11: e0167809. https://doi.org/10.1371/journal.pone.0167809

Costanza R., de Groot R., Sutton P., et al. 2014. Changes in the global value of ecosystem services. Global Environ. Chang. 26: 152-158. https://doi.org/10.1016/j.gloenvcha.2014.04.002

Costello M.J., Coll M., Danovaro R., et al. 2010. A census of marine biodiversity knowledge, resources, and future challenges. PloS ONE 5: e12110. https://doi.org/10.1371/journal.pone.0012110

Dufour F., Guidetti P., Francour P. 2007. Comparison of fish inventory in Mediterranean marine protected areas: Influence of surface area and age. Cybium 31: 19-31

Elliott M., Hemingway K., Marshall S., et al. 2002. Data quality analysis and interpretation. In: Elliott M., Hemmingway K.L. (eds), Fishes in Estuaries. Blackwell Science, Oxford, pp. 510-554. https://doi.org/10.1002/9780470995228

Fairclough D., Brown J., Carlish B., et al. 2014. Breathing life into fisheries stock assessments with citizen science. Sci. Rep. 4: 7249 https://doi.org/10.1038/srep07249

Franco A., Pérez-Ruzafa A., Drouineau H., et al. 2012. Assessment of fish assemblages in coastal lagoon habitats: Effect of sampling method. Estuar. Coast. Shelf Sci. 112: 115-125. https://doi.org/10.1016/j.ecss.2011.08.015

Francour P. 1994. Pluriannual analysis of the reserve effect on ichthyofauna in the Scandola natural reserve (Corsica, Northwestern Mediterranean). Oceanol. Acta 17: 309-317.

Francour P. 1999. A critical review of adult and juvenile fish sampling techniques in Posidonia oceanica seagrass beds. Nat. Sicil. 23: 33-57.

Fraschetti S., Guarnieri G., Bevilacqua S., et al. 2011. Conservation of Mediterranean habitats and biodiversity countdowns: what information do we really need? Aquat. Conserv. 21: 299-306. https://doi.org/10.1002/aqc.1185

García-Rubies A., Zabala M. 1990. Effects of total fishing prohibition on the rocky fish assemblages of Medes Islands marine reserve (NW Mediterranean). Sci. Mar. 54: 317-328.

García-Rubies A., Hereu B., Zabala M. 2013. Long-Term Recovery Patterns and Limited Spillover of Large Predatory Fish in a Mediterranean MPA. PloS ONE 8: e73922. https://doi.org/10.1371/journal.pone.0073922

Glavičić I., Paliska D., Soldo A., et al. 2016. A quantitative assessment of the cryptobenthic fish assemblage at deep littoral cliffs in the Mediterranean. Sci. Mar. 80: 329-337. https://doi.org/10.3989/scimar.04307.23A

Gledhill C.T., Lyczkowski-Shultz J., Rademacher K., et al. 1996. Evaluation of video and acoustic index methods for assessing reef-fish populations. ICES J. Mar. Sci. 53: 483-485 https://doi.org/10.1006/jmsc.1996.0069

Gordoa A. 2009. Characterization of the infralittoral system along the north-east Spanish coast based on sport shore-based fishing tournament catches. Estuar. Coast. Shelf Sci. 82: 41-49. https://doi.org/10.1016/j.ecss.2008.11.024

Guidetti P., Fanelli G., Fraschetti S., et al. 2002. Coastal fish indicate human-induced changes in the Mediterranean littoral. Mar. Environ. Res. 53: 77-94. https://doi.org/10.1016/S0141-1136(01)00111-8

Guilhaumon F., Albouy C., Claudet J., et al. 2015. Representing taxonomic, phylogenetic and functional diversity: new challenges for Mediterranean marine protected areas. Divers. Distrib. 21: $175-187$. https://doi.org/10.1111/ddi.12280

Halpern B.S., Warner R.R. 2003. Review paper. Matching marine reserve design to reserve objectives. Proc. R. Soc. B 270: 1871-1878.

Harmelin-Vivien M., Harmelin J., Chauvet C., et al. 1985. The underwater observation of fish communities and fish populations. Methods and problems. Rev. Ecol. Terr. Vie 40: 466-539.

Harmelin-Vivien M., Le Diréach L., Bayle-Sempere J., et al. 2008. Gradients of abundance and biomass across reserve boundaries in six Mediterranean marine protected areas: Evidence of fish spillover? Biol. Conserv. 141: 1829-1839. https://doi.org/10.1016/j.biocon.2008.04.029

Holmlund C.M., Hammer M. 1999. Ecosystem services generated by fish populations. Ecol. Econ. 29: 253-268. https://doi.org/10.1016/S0921-8009(99)00015-4 
Hutchings J.A., Baum J.K. 2005. Measuring marine fish biodiversity: temporal changes in abundance, life history and demography. Philos. Trans. R. Soc. B 360: 315-338. https://doi.org/10.1098/rstb.2004.1586

Illich I.P., Kotrschal K. 1990. Depth distribution and abundance of Northern Adriatic littoral rocky reef blennioid fishes (Blennidae and Trypterygion). Mar. Ecol. 11: 277-289. https://doi.org/10.1111/j.1439-0485.1990.tb00384.x

Kotrschal K. 1988. Blennies and endolithic bivalves: differential utilization of shelter in Adriatic Blenniidae (Pisces: Teleostei). Mar. Ecol. 9: 253-269. https://doi.org/10.1111/j.1439-0485 1988.tb00332.x

Kovačić M., Patzner R.A., Schliewen U. 2012. A first quantitative assessment of the ecology of cryptobenthic fishes in the Mediterranean Sea. Mar. Biol. 159: 2731-2742. https://doi.org/10.1007/s00227-012-2030-6

Kovačić M., Sanda R. 2016. A new species of Gobius (Perciformes: Gobiidae) from the Mediterranean Sea and the redescription of Gobius bucchichi. J. Fish Biol. 88: 1104-1124. https://doi.org/10.1111/jfb.12883

La Mesa G., Vacchi M. 1999. An analysis of the coastal fish assemblage of the Ustica Island Marine Reserve (Mediterranean Sea). Mar. Ecol. 20: 147-165. https://doi.org/10.1046/j.1439-0485.1999.00067.x

La Mesa G., Micalizzi M., Giaccone G., et al. 2004. Cryptobenthic fishes of the Ciclopi Islands marine reserve (central Mediterranean Sea): assemblage composition, structure and relations with habitat features. Mar. Biol. 145: 233-242. https://doi.org/10.1007/s00227-004-1315-9

La Mesa G., Di Muccio S., Vacchi M. 2006. Structure of a Mediterranean cryptobenthic fish community and its relationships with habitat characteristics. Mar. Biol. 149: 149-167. https://doi.org/10.1007/s00227-005-0194-Z

La Mesa G., Molinari A., Tunesi L. 2010. Coastal fish assemblage characterisation to support the zoning of a new Marine Protected Area in north-western Mediterranean. Ital. J. Zool. 77: 197-210. https://doi.org/10.1080/11250000903413668

Lester S.E., Halpern B.S., Grorud-Colvert K., et al. 2009. Biological effects within no-take marine reserves: a global synthesis. Mar. Ecol. Prog. Ser. 384: 33-46. https://doi.org/10.3354/meps08029

Lincoln Smith M.P. 1989. Improving multispecies rocky reef fish censuses by counting different groups of species using different procedures. Environ. Biol. Fish. 26: 29-37. https://doi.org/10.1007/BF00002473

Lotze H.K., Lenihan H.S., Bourque B.J., et al. 2006. Depletion, degradation, and recovery potential of estuaries and coastal seas. Science 312: 1806-1809. https://doi.org/10.1126/science.1128035

MacNeil M.A., Tyler E.H., Fonnesbeck C.J., et al. 2008. Accounting for detectability in reef-fish biodiversity estimates. Mar. Ecol. Prog. Ser. 367: 249-260. https://doi.org/10.3354/meps07580

Macpherson E. 1994. Substrate utilization in a Mediterranean littoral fish community. Mar. Ecol. Prog. Ser. 114: 211-218. https://doi.org/10.3354/meps114211

Mallet D., Pelletier D. 2014. Underwater video techniques for observing coastal marine biodiversity: a review of sixty years of publications (1952-2012). Fish. Res. 154: 44-62. https://doi.org/10.1016/j.fishres.2014.01.019

Myers R.A., Baum J.K., Shepherd T.D., et al. 2007. Cascading effects of the loss of apex predatory sharks from a coastal ocean. Science 315: 1846-1850. https://doi.org/10.1126/science.1138657

Ordines F., Massuti E. 2009. Relationships between macro-epibenthic communities and fish on the shelf grounds of the western Mediterranean. Aquat. Conserv. 19: 370-383. https://doi.org/10.1002/aqc.969

Patzner R.A. 1999. Habitat utilization and depth distribution of small cryptobenthic fishes (Blenniidae, Gobiesocidae, Gobiidae, Tripterygiidae) in Ibiza (western Mediterranean Sea). Environ. Biol. Fish. 55: 207-214. https://doi.org/10.1023/A:1007535808710

Petrakis G., Stergiou K. 1995. Weight-length relationships for 33 fish species in Greek waters. Fish. Res. 21: 465-469. https://doi.org/10.1016/0165-7836(94)00294-7

Pickaver A.H. 2010. 10 Integrated coastal zone management progress and sustainability indicators. In: Telford T. (eds), Integrated Coastal Zone Management. Wiley-Blackwell, Oxford, UK. pp 226-250. https://doi.org/10.1680/czm.35164.0010

Prato G, Thiriet P. Di Franco A, et al. 2017. Enhancing fish Underwater Visual Census to move forward assessment of fish assemblages: An application in three Mediterranean Marine Protected Areas. PLoS ONE 12: e0178511.

https://doi.org/10.1371/journal.pone.0178511

R Development Core Team. 2013. R: A language and environment for statistical computing. R Foundation for Statistical Computing, Vienna, Austria. http://www.R-project.org

Rousseeuw P.J. 1987. Silhouettes: A graphical aid to the interpretation and validation of cluster analysis. J. Comput. Appl. Math. 20: 53-65. https://doi.org/10.1016/0377-0427(87)90125-7

Sahyoun R., Bussotti S., Di Franco A., et al. 2013. Protection effects on Mediterranean fish assemblages associated with different rocky habitats. J. Mar. Biol. Assoc. UK 93: 425-435. https://doi.org/10.1017/S0025315412000975

Sale P.F., Douglas W.A. 1981. Precision and accuracy of visual census technique for fish assemblages on coral patch reefs. Environ. Biol. Fish. 6: 333-339. https://doi.org/10.1007/BF00005761

Sano M. 2000. Stability of reef fish assemblages: responses to coral recovery after catastrophic predation by Acanthaster planci. Mar. Ecol. Prog. Ser. 198: 121-130. https://doi.org/10.3354/meps 198121

Schindler D.E., Carpenter S.R., Cole J.J., et al. 1997. Influence of food web structure on carbon exchange between lakes and the atmosphere. Science 277: 248-251. https://doi.org/10.1126/science.277.5323.248

Seytre C., Francour P. 2008. Is the Cape Roux marine protected area (Saint-Raphaël, Mediterranean Sea) an efficient tool to sustain artisanal fisheries? First indications from visual censuses and trammel net sampling. Aquat. Living Resour. 21: 297-305. https://doi.org/10.1051/alr:2008043

Sheldon A.L. 1988. Conservation of stream fishes: patterns of diversity, rarity, and risk. Cons. Biol. 2: 149-156. https://doi.org/10.1111/j.1523-1739.1988.tb00166.x

Smith M.L. 1988. Effects of observer swimming speed on sample counts of temperate rocky reef fish assemblages. Mar. Ecol. Prog. Ser. 43: 223-231. https://doi.org/10.3354/meps043223

Smith-Vaniz W.F., Jelks H.L., Rocha L.A. 2006. Relevance of cryptic fishes in biodiversity assessments: a case study at Buck Island Reef National Monument, St. Croix. Bull. Mar. Sci. 79: 17-48.

Stobart B., García-Charton J.A., Espejo C., et al. 2007. A baited underwater video technique to assess shallow-water Mediterranean fish assemblages: Methodological evaluation. J. Exp. Mar. Biol. Ecol. 345: 158-174 https://doi.org/10.1016/j.jembe.2007.02.009

Støttrup J.G. 2009. The challenge towards sustainable utilisation of coastal fish resources. In: Moksness E., Dahl E., Støttrup J. (eds), Integrated Coastal Zone Management. John Wiley \& Sons, pp. 25-34 https://doi.org/10.1002/9781444316285.ch2

Tessier E., Chabanet P. 2006. Using video techniques for estimating fish post-larvae abundance after mass settlement on artificial reefs. Proc. 10th Intl. Coral Reef Symp., Okinawa, Japan.

Tessier A., Pastor J., Francour P., et al. 2013. Video transects as a complement to underwater visual census to study reserve effect on fish assemblages. Aquat. Biol. 18: 229-241. https://doi.org/10.3354/ab00506

Tiralongo F., Tibullo D., Brundo M.V., et al. 2016. Habitat preference of combtooth blennies (Actinopterygii: Perciformes: Blenniidae) in very shallow waters of the Ionian Sea, South-Eastern Sicily, Italy. Acta Ichthyol. Piscat. 46: 65-75. https://doi.org/10.3750/AIP2016.46.2.02

Thiriet P.D., Di Franco A., Cheminée A., et al. 2016. Abundance and Diversity of Crypto-and Necto-Benthic Coastal Fish Are Higher in Marine Forests than in Structurally Less Complex Macroalgal Assemblages. PloS ONE 11: e0164121. https://doi.org/10.1371/journal.pone.0164121

Vanni M.J. 2002. Nutrient cycling by animals in freshwater ecosystems. Annu. Rev. Ecol. Syst. 33: 341-370. https://doi.org/10.1146/annurev.ecolsys.33.010802.150519

Willis T.J. 2001. Visual census methods underestimate density and diversity of cryptic reef fishes. J. Fish Biol. 59: 1408-1411. https://doi.org/10.1111/j.1095-8649.2001.tb00202.x

Willis T.J., Anderson M.J. 2003. Structure of cryptic reef fish assemblages: relationships with habitat characteristics and preda- 
tor density. Mar. Ecol. Prog. Ser. 257: 209-221. https://doi.org/10.3354/meps257209

Willis T.J., Babcock R.C. 2000. A baited underwater video system for the determination of relative density of carnivorous reef fish. Mar. Freshwater Res. 51: 755-763. https://doi.org/10.1071/MF00010

Willis T.J., Millar R.B., Babcock R.C. 2000. Detection of spatial variability in relative density of fishes: comparison of visual census, angling, and baited underwater video. Mar. Ecol. Prog. Ser. 198: 249-260.

https://doi.org/10.3354/meps198249

Winer B.J., Broan D.R., Michels K.M. 1991. Statistical Principles in Experimental Design. McGraw-Hill, New York.

Zander C., Heymer A. 1970. Tripterygion tripteronotus (Risso, 1810) and Tripterygion xanthosoma $\mathrm{n}$ sp., an ecological speciation (Pisces, Teleostei). Vie Milieu 21: 363-394. 